\title{
Superconductivity from purely repulsive interactions in the strong coupling approach : Application of the SU(2) slave-rotor theory to the Hubbard model
}

\author{
Ki-Seok Kim ${ }^{1}$ and Mun Dae Kim² \\ ${ }^{1}$ Asia Pacific Center for Theoretical Physics, Hogil Kim Memorial building 5th floor, \\ POSTECH, Hyoja-dong, Namgu, Pohang 790-784, Korea \\ ${ }^{2}$ Institute of Physics and Applied Physics, Yonsei University, Seoul 120-749, Korea
}

(Dated: December 4, 2018)

\begin{abstract}
We propose a mechanism of superconductivity from purely repulsive interactions in the strong coupling regime, where the BCS (Bardeen-Cooper-Schrieffer) mechanism such as the spin-fluctuation approach is difficult to apply. Based on the SU(2) slave-rotor representation of the Hubbard model, we find that the single energy scale for the amplitude formation of Cooper pairs and their phase coherence is separated into two energy scales, allowing the so called pseudogap state where such Cooper pairs are coherent locally but not globally, interpreted as realization of the density-phase uncertainty principle. This superconducting state shows the temperature-linear decreasing ratio of superfluid weight, resulting from strong phase fluctuations.

PACS numbers: 71.10.-w, 71.10.Hf, 74.20.-z, 74.20.Mn
\end{abstract}

\section{INTRODUCTION}

To find the mechanism of superconductivity from purely repulsive interactions has been one of the central interests during the last two decades associated with high $T_{c}$ cuprates [1]. It was shown that purely repulsive interactions can turn into attractive ones via some renormalization processes associated with spin-density-wave fluctuations, if the Fermi-surface topology has a special nesting structure as the case of high $\mathrm{T}_{c}$ cuprates [2] or Fe-based superconductors [3], for example, described by the so called spin-fluctuation approach. In this approach phonons are replaced with antiferromagnetic fluctuations taking the role of pairing glue [4], thus basically the same as the strong coupling BCS (Bardeen-Cooper-Schrieffer) theory in the Eliashberg approximation [5]. Unfortunately, such an approach loses its theoretical validity in the strong coupling regime because the spin-fermion model itself and its evaluation way are justified only in the weak coupling limit [6], i.e., $U / D \ll 1$ with the interaction strength $U$ and half bandwidth $D$.

This reminds us of two kinds of theories for magnetism; the Hertz-Moriya-Millis (HMM) theory is the standard framework for itinerant electrons [7] while the magnetism from localized spins is successfully described by the Schwinger-boson gauge theory [8]. Importantly, the strong coupling approach of the Schwinger-boson theory gives rise to two kinds of energy scales, associated with formation of short range antiferromagnetic correlations and long range ordering for antiferromagnetism. Emergence of the spin-gapped state above an antiferromagnetic order reflects strong quantum fluctuations of spin dynamics, guaranteed by the uncertainty relation of spins.

In this paper we propose a mechanism of superconductivity in the strong coupling regime. Recently, one of us has suggested an SU(2) slave-rotor representation of the Hubbard model, where not only local density fluctuations but also on-site pairing excitations are taken into account on equal footing, giving rise to superconducting fluctuations naturally [9]. We here obtain an effective U(1) gauge theory called the pair-rotor theory of the Hubbard model, where phase fluctuations of Cooper pairs are extracted from the $\mathrm{SU}(2)$ slave-rotor description. We show that the single energy scale for the amplitude formation of Cooper pairs and their phase coherence is separated into two energy scales, allowing the so called pseudogap state where the Cooper pairs are coherent locally but not globally. One can say that this superconducting state resembles the RVB (resonating-valance-bond) superconductivity [10]. However the present mechanism is of charge-fluctuation-induced, while for the RVB the slaveboson study of the t-J model [11] is of spin-fluctuationinduced, where charge fluctuations are completely frozen out.

Superconductivity of the U(1) pair-rotor theory is analogous with antiferromagnetism of the Schwingerboson theory, where the amplitude formation of Cooper pairs and their global phase coherence correspond to short range antiferromagnetic correlations and condensation of Schwinger bosons, respectively, and the densityphase uncertainty matches with the uncertainty relation between spins. In this respect the pseudogap state of the pair-rotor theory agrees with the spin-gapped phase of the Schwinger-boson theory, identified with the hallmark of the strong coupling approach. On the other hand, this description should be differentiated from the $\mathrm{U}(1)$ slave-rotor theory of the t-J-U model where d-wave singlet pairs originate from the spin-exchange term, but the $\mathrm{U}(1)$ slave-rotor field has nothing to do with pairing fluctuations [9].

We would like to point out an interesting reformulation of the Hubbard model, where the role of on-site pairing fluctuations is emphasized [12]. Although this formulation differs from the present $\mathrm{SU}(2)$ slave-rotor theory which is a gauge theory, it also suggests that such quantum fluctuations give rise to superconducting correlations 
in doped Mott insulators.

\section{SU(2) SLAVE-ROTOR REPRESENTATION OF THE HUBBARD MODEL}

We rewrite the Hubbard model

$$
H=-t \sum_{\langle i j\rangle}\left(c_{i \sigma}^{\dagger} e^{i A_{i j}} c_{j \sigma}+H . c .\right)+\frac{3 u}{2} \sum_{i} c_{i \uparrow}^{\dagger} c_{i \uparrow} c_{i \downarrow}^{\dagger} c_{i \downarrow}
$$

as follows [9],

$$
\begin{aligned}
& Z=\int D\left[\eta_{i}, U_{i}, \vec{\Omega}_{i}, E_{i j}, F_{i j}\right] e^{-\int_{0}^{\beta} d \tau L}, \\
& L=L_{0}+L_{\eta}+L_{U}, \quad L_{0}=t \sum_{\langle i j\rangle} \operatorname{tr}\left(F_{i j} E_{i j}^{\dagger}+H . c .\right), \\
& L_{\eta}=\sum_{i} \eta_{i}^{\dagger}\left(\partial_{\tau} \mathbf{I}-i \vec{\Omega}_{i} \cdot \vec{\tau}\right) \eta_{i}-t \sum_{\langle i j\rangle}\left(\eta_{i}^{\dagger} F_{i j} \eta_{j}+H . c .\right), \\
& L_{U}=\frac{1}{4 u} \sum_{i} \operatorname{tr}\left(-i U_{i} \partial_{\tau} U_{i}^{\dagger}+\vec{\Omega}_{i} \cdot \vec{\tau}+i \mu U_{i} \tau_{3} U_{i}^{\dagger}\right)^{2} \\
& -t \sum_{\langle i j\rangle} \operatorname{tr}\left(U_{j}^{\dagger} E_{i j}^{\dagger} U_{i} e^{i A_{i j} \tau_{3}} \tau_{3}+H . c .\right)
\end{aligned}
$$

where an electron $\Phi_{i}=\left(\begin{array}{c}c_{i \uparrow} \\ c_{i \downarrow}^{\dagger}\end{array}\right)$ is assumed to be a composite of a holon $U_{i}=\left(\begin{array}{cc}z_{i \uparrow} & -z_{i \downarrow}^{\dagger} \\ z_{i \downarrow} & z_{i \uparrow}^{\dagger}\end{array}\right)$ and a spinon $\eta_{i}=\left(\begin{array}{c}\eta_{i+} \\ \eta_{i-}^{\dagger}\end{array}\right)$ carrying charge and spin quantum numbers, respectively, given by

$$
\Phi_{i}=U_{i}^{\dagger} \eta_{i}
$$

with the constraint $\left|z_{i \uparrow}\right|^{2}+\left|z_{i \downarrow}\right|^{2}=1 . E_{i j}$ and $F_{i j}$ are $2 \times 2$ matrix fields associated with hopping of holons and spinons, respectively, and $\vec{\Omega}_{i}$ is an isospin field related with on-site density and pairing potentials. $\mu$ is an electron chemical potential, and $A_{i j}$ is an external electromagnetic field.

It is not difficult to see equivalence between the $\mathrm{SU}(2)$ slave-rotor effective Lagrangian [Eq. (2)] and Hubbard model [Eq. (1)], integrating over field variables of $E_{i j}$, $F_{i j}$ and $\vec{\Omega}_{i}$ and replacing the composite field $U_{i}^{\dagger} \eta_{i}$ with an electron field $\Phi_{i}$. The procedure is well described in the previous study [9]. An important feature in the $\mathrm{SU}(2)$ slave-rotor description is the emergence of pairing correlations between nearest neighbor electrons, given by off diagonal hopping in $F_{i j}$ which results from on-site pairing fluctuations, captured by the off diagonal variable $z_{i \downarrow}$ of the $\mathrm{SU}(2)$ matrix field $U_{i}$. However, the appearance of pairing correlations does not necessarily lead to superconductivity because their global coherence, described by condensation of $\mathrm{SU}(2)$ matrix holons, is not guaranteed.
The similar situation happens in the $\mathrm{SU}(2)$ slave-boson theory of the t-J model [11].

We write $\mathrm{SU}(2)$ hopping matrices as $E_{i j} \approx E W_{i j} \tau_{3}$ and $F_{i j} \approx F W_{i j} \tau_{3}$, where their amplitudes are assumed to be homogeneous and the $\mathrm{SU}(2)$ phase factor can be represented as

$$
W_{i j} \equiv\left(\begin{array}{cc}
X_{i j} & -Y_{i j}^{\dagger} \\
Y_{i j} & X_{i j}^{\dagger}
\end{array}\right)
$$

without losing generality, satisfying the unitary constraint $\left|X_{i j}\right|^{2}+\left|Y_{i j}\right|^{2}=1$. Then, we find an effective $\mathrm{SU}(2)$ slave-rotor action

$$
\begin{aligned}
& Z=\int D\left[\eta_{i s}, z_{i \sigma}, \vec{\Omega}_{i}, X_{i j}, Y_{i j}\right] \delta\left(\left|X_{i j}\right|^{2}+\left|Y_{i j}\right|^{2}-1\right) \\
& \delta\left(\left|z_{i \uparrow}\right|^{2}+\left|z_{i \downarrow}\right|^{2}-1\right) \exp \left(-\int_{0}^{\beta} d \tau L\right) \\
& L=L_{\eta}+L_{U}+4 t \sum_{\langle i j\rangle} E F, \\
& L_{\eta}=\sum_{i}\left(\begin{array}{ll}
\eta_{i+}^{\dagger} & \eta_{i-}
\end{array}\right)\left(\begin{array}{cc}
\partial_{\tau}-i \Omega_{i}^{z} & -i\left[\Omega_{i}^{x}-i \Omega_{i}^{y}\right] \\
-i\left[\Omega_{i}^{x}+i \Omega_{i}^{y}\right] & \partial_{\tau}+i \Omega_{i}^{z}
\end{array}\right) \\
& \left(\begin{array}{c}
\eta_{i+} \\
\eta_{i-}^{\dagger}
\end{array}\right)-t F \sum_{\langle i j\rangle}\left\{\left(\begin{array}{ll}
\eta_{i+}^{\dagger} & \eta_{i-}
\end{array}\right)\left(\begin{array}{cc}
X_{i j} & Y_{i j}^{\dagger} \\
Y_{i j} & -X_{i j}^{\dagger}
\end{array}\right)\left(\begin{array}{c}
\eta_{j+} \\
\eta_{j-}^{\dagger}
\end{array}\right)\right. \\
& +H . c .\} \text {, } \\
& L_{U}=\frac{1}{4 u} \sum_{i}\left\{\left(-i\left[z_{i \uparrow} \partial_{\tau} z_{i \uparrow}^{\dagger}+z_{i \downarrow}^{\dagger} \partial_{\tau} z_{i \downarrow}\right]+\Omega_{i}^{z}\right.\right. \\
& \left.+i \mu\left(\left|z_{i \uparrow}\right|^{2}-\left|z_{i \downarrow}\right|^{2}+1\right)\right)^{2}+\left(-i\left[z_{i \uparrow} \partial_{\tau} z_{i \downarrow}^{\dagger}-z_{i \downarrow}^{\dagger} \partial_{\tau} z_{i \uparrow}\right]\right. \\
& \left.+\Omega_{i}^{x}-i \Omega_{i}^{y}+2 i \mu z_{i \uparrow} z_{i \downarrow}^{\dagger}\right)\left(-i\left[z_{i \downarrow} \partial_{\tau} z_{i \uparrow}^{\dagger}-z_{i \uparrow}^{\dagger} \partial_{\tau} z_{i \downarrow}\right]\right. \\
& \left.+\Omega_{i}^{x}+i \Omega_{i}^{y}+2 i \mu z_{i \uparrow}^{\dagger} z_{i \downarrow}\right)+\left(-i\left[z_{i \downarrow} \partial_{\tau} z_{i \uparrow}^{\dagger}-z_{i \uparrow}^{\dagger} \partial_{\tau} z_{i \downarrow}\right]\right. \\
& \left.+\Omega_{i}^{x}+i \Omega_{i}^{y}+2 i \mu z_{i \uparrow}^{\dagger} z_{i \downarrow}\right)\left(-i\left[z_{i \uparrow} \partial_{\tau} z_{i \downarrow}^{\dagger}-z_{i \downarrow}^{\dagger} \partial_{\tau} z_{i \uparrow}\right]\right. \\
& \left.+\Omega_{i}^{x}-i \Omega_{i}^{y}+2 i \mu z_{i \uparrow} z_{i \downarrow}^{\dagger}\right)+\left(-i\left[z_{i \downarrow} \partial_{\tau} z_{i \downarrow}^{\dagger}+z_{i \uparrow}^{\dagger} \partial_{\tau} z_{i \uparrow}\right]\right. \\
& \left.\left.-\Omega_{i}^{z}-i \mu\left(\left|z_{i \uparrow}\right|^{2}-\left|z_{i \downarrow}\right|^{2}+1\right)\right)^{2}\right\} \\
& -2 t E \sum_{\langle i j\rangle}\left\{\left(\begin{array}{cc}
z_{i \uparrow}^{\dagger} & z_{i \downarrow}^{\dagger}
\end{array}\right)\left(\begin{array}{cc}
X_{i j} e^{-i A_{i j}} & Y_{i j}^{\dagger} e^{-i A_{i j}} \\
Y_{i j} e^{-i A_{i j}} & -X_{i j}^{\dagger} e^{-i A_{i j}}
\end{array}\right)\right. \\
& \left.\left(\begin{array}{c}
z_{j \uparrow} \\
z_{j \downarrow}
\end{array}\right)+H . c .\right\} .
\end{aligned}
$$

Our main problem is how to extract dynamics for phase fluctuations of pairing order parameters from the boson sector of the $\mathrm{SU}(2)$ slave-rotor theory. The easy axis approximation of $U_{i}=e^{i \phi_{i} \tau_{3}}$ implying $z_{i \downarrow}=0$ does not allow pairing correlations, identified with on-site density fluctuations and giving rise to the Mott transition from a paramagnetic Mott insulator to a Fermi liquid metal via their condensation [13]. In this study we take an easy 
plane limit, introducing pairing correlations. Justification of this approximation can be given in the similar way as the $\mathrm{SU}(2)$ slave-boson theory [11].

\section{U(1)-PAIR SLAVE-ROTOR THEORY}

\section{A. Easy plane approximation}

We introduce an isospin field

$$
\overrightarrow{I_{i}} \equiv \frac{1}{2} z_{i \sigma}^{\dagger} \vec{\tau}_{\sigma \sigma^{\prime}} z_{i \sigma^{\prime}},
$$

and consider an easy plane limit

$$
\vec{I}_{i}=I_{i}^{x} \hat{x}+I_{i}^{y} \hat{y}
$$

with $I_{i}^{x 2}+I_{i}^{y 2}=1 / 2$, described by

$$
z_{i \uparrow}=\frac{1}{\sqrt{2}} e^{i \phi_{i \uparrow}}, \quad z_{i \downarrow}=\frac{1}{\sqrt{2}} e^{i \phi_{i \downarrow}} .
$$

Inserting Eq. (5) into Eq. (4), we find

$$
\begin{aligned}
& L_{\phi}=\frac{1}{2 u} \sum_{i}\left\{\left(\frac{1}{2}\left[\partial_{\tau} \phi_{i \uparrow}-\partial_{\tau} \phi_{i \downarrow}\right]-\Omega_{i}^{z}-i \mu\right)^{2}\right. \\
& +\left(\frac{1}{2}\left[\partial_{\tau} \phi_{i \uparrow}+\partial_{\tau} \phi_{i \downarrow}\right]-\left[\Omega_{i}^{x}+i \Omega_{i}^{y}\right] e^{i\left(\phi_{i \uparrow}-\phi_{i \downarrow}\right)}-i \mu\right) \\
& \left.\left(\frac{1}{2}\left[\partial_{\tau} \phi_{i \uparrow}+\partial_{\tau} \phi_{i \downarrow}\right]-\left[\Omega_{i}^{x}-i \Omega_{i}^{y}\right] e^{-i\left(\phi_{i \uparrow}-\phi_{i \downarrow}\right)}-i \mu\right)\right\} \\
& -t E \sum_{\langle i j\rangle}\left\{\left(e^{-i \phi_{i \uparrow}} e^{-i \phi_{i \downarrow}}\right)\left(\begin{array}{cc}
X_{i j} e^{-i A_{i j}} & Y_{i j}^{\dagger} e^{-i A_{i j}} \\
Y_{i j} e^{-i A_{i j}} & -X_{i j}^{\dagger} e^{-i A_{i j}}
\end{array}\right)\right. \\
& \left.\left(\begin{array}{c}
e^{i \phi_{j \uparrow}} \\
e^{i \phi_{j \downarrow}}
\end{array}\right)+H . c .\right\},
\end{aligned}
$$

where the spinon part is the same as that of Eq. (4). As shown in this effective theory, the presence of the off diagonal term in the kinetic energy of rotorons allows us to control global phase coherence of spinon-pairing excitations. We note that this effective Lagrangian is analogous with that of the $d$-wave pairing state in the $\mathrm{SU}(2)$ slaveboson theory, where gauge fluctuations may give rise to composite pairing fluctuations between different boson species [11], corresponding to $\left(\phi_{i \uparrow}+\phi_{i \downarrow}\right) / 2$ in the $\mathrm{U}(1)$ pair-rotor theory.

The isospin field was argued to prefer an easy plane in the non-linear $\sigma$ model description of the $\mathrm{SU}(2)$ slaveboson theory when holes are doped, resulting from an effective potential for the easy plane anisotropy [11]. Even if the easy plane approximation is difficult to justify selfconsistently, the present formulation gives us a chance to investigate the role of pairing fluctuations beyond the conventional description.

\section{B. Gauge transformation}

\section{Spinon sector}

One can make the phase factor gauged away in the phase-gauge coupling term of Eq. (6), performing the gauge transformation

$$
\begin{aligned}
& \Omega_{i}^{x}-i \Omega_{i}^{y} \rightarrow\left(\Omega_{i}^{x}-i \Omega_{i}^{y}\right) e^{i\left(\phi_{i \uparrow}-\phi_{i \downarrow}\right)}, \\
& \Omega_{i}^{x}+i \Omega_{i}^{y} \rightarrow\left(\Omega_{i}^{x}+i \Omega_{i}^{y}\right) e^{-i\left(\phi_{i \uparrow}-\phi_{i \downarrow}\right)} .
\end{aligned}
$$

Then, the spinon Lagrangian in Eq. (4) is given by

$$
\begin{aligned}
& L_{\eta}=\sum_{i}\left(\eta_{i+}^{\dagger} \eta_{i-}\right) \\
& \left(\begin{array}{cc}
\partial_{\tau}-i \Omega_{i}^{z} & -i\left(\Omega_{i}^{x}-i \Omega_{i}^{y}\right) e^{i\left(\phi_{i \uparrow}-\phi_{i \downarrow}\right)} \\
-i\left(\Omega_{i}^{x}+i \Omega_{i}^{y}\right) e^{-i\left(\phi_{i \uparrow}-\phi_{i \downarrow}\right)} & \partial_{\tau}+i \Omega_{i}^{z}
\end{array}\right) \\
& \left(\begin{array}{c}
\eta_{i+} \\
\eta_{i-}^{\dagger}
\end{array}\right)-t F \sum_{\langle i j\rangle}\left\{\left(\begin{array}{ll}
\eta_{i+}^{\dagger} & \eta_{i-}
\end{array}\right)\left(\begin{array}{cc}
X_{i j} & Y_{i j}^{\dagger} \\
Y_{i j} & -X_{i j}^{\dagger}
\end{array}\right)\left(\begin{array}{c}
\eta_{j+} \\
\eta_{j-}^{\dagger}
\end{array}\right)\right. \\
& + \text { H.c. }\} .
\end{aligned}
$$

To make the phase factor gauged away in the off diagonal part of the spinon Lagrangian, we introduce the gauge transformation of

$$
\begin{aligned}
& \psi_{i \uparrow}=e^{-i\left(\phi_{i \uparrow}-\phi_{i \downarrow}\right) / 2} \eta_{i+}, \\
& \psi_{i \downarrow}^{\dagger}=e^{i\left(\phi_{i \uparrow}-\phi_{i \downarrow}\right) / 2} \eta_{i-}^{\dagger},
\end{aligned}
$$

where $\psi_{i \sigma}$ is a renormalized spinon via virtual pairing fluctuations. Then, the phase field appears in the time derivative and $\mathrm{SU}(2)$ gauge matrix $W_{i j}$. Considering the gauge transformation

$$
\begin{aligned}
\Omega_{i}^{z} & \rightarrow \Omega_{i}^{z}+\frac{1}{2}\left(\partial_{\tau} \phi_{i \uparrow}-\partial_{\tau} \phi_{i \downarrow}\right), \\
X_{i j} & \rightarrow e^{i\left(\phi_{i \uparrow}-\phi_{i \downarrow}\right) / 2} X_{i j} e^{-i\left(\phi_{j \uparrow}-\phi_{j \downarrow}\right) / 2}, \\
Y_{i j} & \rightarrow e^{-i\left(\phi_{i \uparrow}-\phi_{i \downarrow}\right) / 2} Y_{i j} e^{-i\left(\phi_{j \uparrow}-\phi_{j \downarrow}\right) / 2},
\end{aligned}
$$

we find an effective Lagrangian for renormalized spinons

$$
\begin{aligned}
& L_{\psi}=\sum_{i}\left(\begin{array}{ll}
\psi_{i \uparrow}^{\dagger} & \psi_{i \downarrow}
\end{array}\right)\left(\begin{array}{cc}
\partial_{\tau}-i \Omega_{i}^{z} & -\left(\Omega_{i}^{x}-i \Omega_{i}^{y}\right) \\
-\left(\Omega_{i}^{x}+i \Omega_{i}^{y}\right) & \partial_{\tau}+i \Omega_{i}^{z}
\end{array}\right) \\
& \left(\begin{array}{c}
\psi_{i \uparrow} \\
\psi_{i \downarrow}^{\dagger}
\end{array}\right)-t F \sum_{\langle i j\rangle}\left\{\left(\begin{array}{ll}
\psi_{i \uparrow}^{\dagger} & \psi_{i \downarrow}
\end{array}\right)\left(\begin{array}{cc}
X_{i j} & Y_{i j}^{\dagger} \\
Y_{i j} & -X_{i j}^{\dagger}
\end{array}\right)\left(\begin{array}{c}
\psi_{j \uparrow} \\
\psi_{j \downarrow}^{\dagger}
\end{array}\right)\right. \\
& + \text { H.c. }\},
\end{aligned}
$$

where the phase field is removed completely. 


\section{Pairon sector}

Based on Eqs. (7) and (9), we obtain

$$
\begin{aligned}
& L_{\phi}=\frac{1}{2 u} \sum_{i}\left\{\left(\Omega_{i}^{z}+i \mu\right)^{2}+\left(\frac{1}{2}\left[\partial_{\tau} \phi_{i \uparrow}+\partial_{\tau} \phi_{i \downarrow}\right]\right.\right. \\
& \left.-\left[\Omega_{i}^{x}+i \Omega_{i}^{y}\right]-i \mu\right)\left(\frac{1}{2}\left[\partial_{\tau} \phi_{i \uparrow}+\partial_{\tau} \phi_{i \downarrow}\right]-\left[\Omega_{i}^{x}-i \Omega_{i}^{y}\right]\right. \\
& -i \mu)\}-2 t E \sum_{\langle i j\rangle}\left\{( Y _ { i j } ^ { \dagger } + Y _ { i j } ) \operatorname { c o s } \left(\frac{\phi_{i \uparrow}+\phi_{i \downarrow}}{2}\right.\right. \\
& \left.-\frac{\phi_{j \uparrow}+\phi_{j \downarrow}}{2}+A_{i j}\right)+i\left(X_{i j}^{\dagger}-X_{i j}\right) \sin \left(\frac{\phi_{i \uparrow}+\phi_{i \downarrow}}{2}\right. \\
& \left.\left.-\frac{\phi_{j \uparrow}+\phi_{j \downarrow}}{2}+A_{i j}\right)\right\} .
\end{aligned}
$$

Introducing new phase variables

$$
\frac{1}{2}\left[\phi_{i \uparrow}+\phi_{i \downarrow}\right] \equiv \phi_{i c}, \quad \frac{1}{2}\left[\phi_{i \uparrow}-\phi_{i \downarrow}\right] \equiv \phi_{i s},
$$

the rotor Lagrangian becomes

$$
\begin{aligned}
& L_{\phi}=\frac{1}{2 u} \sum_{i}\left\{\left(\Omega_{i}^{z}+i \mu\right)^{2}\right. \\
& \left.+\left(\partial_{\tau} \phi_{i c}-\left[\Omega_{i}^{x}+i \Omega_{i}^{y}\right]-i \mu\right)\left(\partial_{\tau} \phi_{i c}-\left[\Omega_{i}^{x}-i \Omega_{i}^{y}\right]-i \mu\right)\right\} \\
& -2 t E \sum_{\langle i j\rangle}\left\{\left(Y_{i j}^{\dagger}+Y_{i j}\right) \cos \left(\phi_{i c}-\phi_{j c}+A_{i j}\right)\right. \\
& \left.+i\left(X_{i j}^{\dagger}-X_{i j}\right) \sin \left(\phi_{i c}-\phi_{j c}+A_{i j}\right)\right\}
\end{aligned}
$$

where anomalous phase-gauge couplings are gauged away. Comparing this pairon Lagrangian with Eq. (6), we see that the phase field of $\phi_{i s}$ disappears via gauge transformation. We call $\phi_{i c}$ pairon because it controls coherence of local singlet pairs, basically the same role as Schwinger-bosons in the Schwinger-boson theory [8].

\section{U(1) pair-rotor effective Lagrangian}

We write down an effective $\mathrm{U}(1)$ pair-rotor theory of the Hubbard model for phase-fluctuating superconduc- tivity

$$
\begin{aligned}
& Z=\int D\left[\psi_{i \sigma}, \phi_{i c}, \vec{\Omega}_{i}, X_{i j}, Y_{i j}\right] \delta\left(\left|X_{i j}\right|^{2}+\left|Y_{i j}\right|^{2}-1\right) \\
& \exp \left(-\int_{0}^{\beta} d \tau L\right), \quad L=L_{\psi}+L_{\phi}+4 t \sum_{\langle i j\rangle} E F \\
& L_{\psi}=\sum_{i} \psi_{i \alpha}^{\dagger}\left(\partial_{\tau} \delta_{\alpha \beta}-i \vec{\Omega}_{i} \cdot \vec{\tau}_{\alpha \beta}\right) \psi_{i \beta} \\
& -t F \sum_{\langle i j\rangle}\left(\psi_{i \alpha}^{\dagger} W_{i j}^{\alpha \beta} \tau_{3} \psi_{j \beta}+H . c .\right), \\
& L_{\phi}=\frac{1}{2 u} \sum_{i}\left(\partial_{\tau} \phi_{i c}-\left[\Omega_{i}^{x}+i \Omega_{i}^{y}\right]-i \mu\right) \\
& \left(\partial_{\tau} \phi_{i c}-\left[\Omega_{i}^{x}-i \Omega_{i}^{y}\right]-i \mu\right) \\
& -2 t E \sum_{\langle i j\rangle}\left\{\left(Y_{i j}^{\dagger}+Y_{i j}\right) \cos \left(\phi_{i c}-\phi_{j c}+A_{i j}\right)\right. \\
& \left.+i\left(X_{i j}^{\dagger}-X_{i j}\right) \sin \left(\phi_{i c}-\phi_{j c}+A_{i j}\right)\right\} \\
& +\frac{1}{2 u} \sum_{i}\left(\Omega_{i}^{z}+i \mu\right)^{2},
\end{aligned}
$$

where superconductivity is characterized by condensation of pairons $\left\langle e^{i \phi_{i c}}\right\rangle \neq 0$ in the presence of pairing correlations, $Y_{i j}$, basically the same as the fact that antiferromagnetism of localized spins is described by condensation of Schwinger-bosons in the presence of local antiferromagnetic correlations. Actually, the pairon field is identified with the phase field of the pairing order parameter, since $Y_{i j}$ plays the role of phase stiffness for $\phi_{i c}$.

An interesting feature of the $\mathrm{U}(1)$ pair-rotor theory is emergence of two energy scales from the single energy scale $u / t$ in the Hubbard model, corresponding to appearance of incoherent singlet correlations and global coherence of such preformed pairs. The former energy scale may be identified with the pseudogap temperature $T^{*}$, and the latter will be the superconducting transition temperature $T_{c}$. Considering that the spinon sector is nothing but the BCS theory in the mean-field approximation, $T^{*}$ is expected to coincide with the mean-field transition temperature of the BCS theory. On the other hand, the pairon Lagrangian corresponds to the XY model in the mean-field approximation, thus $T_{c}$ will be the coherence temperature of the XY model.

\section{Electron as a composite of spinon and pairon}

An electron field can be represented as a composite object of a spinon and a pairon,

$$
\left(\begin{array}{c}
c_{i \uparrow} \\
c_{i \downarrow}^{\dagger}
\end{array}\right)=\frac{1}{\sqrt{2}}\left(\begin{array}{cc}
e^{-i \phi_{i c}} & e^{-i \phi_{i c}} \\
-e^{i \phi_{i c}} & e^{i \phi_{i c}}
\end{array}\right)\left(\begin{array}{c}
\psi_{i \uparrow} \\
\psi_{i \downarrow}^{\dagger}
\end{array}\right),
$$

where condensation of pairons $\left\langle e^{-i \phi_{i c}}\right\rangle \neq 0$ recovers the BCS quasiparticle relation, allowing superconductivity. 
Inserting the U(1) pair-rotor representation [Eq. (12)] into the Hubbard model [Eq. (1)], one can obtain the $\mathrm{U}(1)$ pair-rotor theory [Eq. (11)] from the Hubbard model directly. The inverse transformation expresses the spinon field in terms of a pairon field and an electron field,

$$
\begin{aligned}
& \psi_{i \uparrow}=\frac{1}{\sqrt{2}} e^{i \phi_{i c}} c_{i \uparrow}-\frac{1}{\sqrt{2}} e^{-i \phi_{i c}} c_{i \downarrow}^{\dagger} \\
& \psi_{i \downarrow}^{\dagger}=\frac{1}{\sqrt{2}} e^{i \phi_{i c}} c_{i \uparrow}+\frac{1}{\sqrt{2}} e^{-i \phi_{i c}} c_{i \downarrow}^{\dagger}
\end{aligned}
$$

Using Eq. (12), one can write down the Cooper pair field as

$$
\begin{aligned}
& \Delta_{i j}^{c p} \equiv\left\langle c_{i \uparrow} c_{j \downarrow}-c_{i \downarrow} c_{j \uparrow}\right\rangle \approx \frac{1}{2}\left\langle e^{-i\left(\phi_{i c}+\phi_{j c}\right)}\right\rangle \\
& \left(\left\langle\psi_{i \uparrow}^{\dagger} \psi_{j \uparrow}+\psi_{j \uparrow}^{\dagger} \psi_{i \uparrow}\right\rangle+\left\langle\psi_{i \downarrow}^{\dagger} \psi_{j \downarrow}+\psi_{j \downarrow}^{\dagger} \psi_{i \downarrow}\right\rangle\right. \\
& \left.+\left\langle\psi_{i \uparrow}^{\dagger} \psi_{j \downarrow}^{\dagger}-\psi_{i \downarrow}^{\dagger} \psi_{j \uparrow}^{\dagger}\right\rangle+\left\langle\psi_{i \uparrow} \psi_{j \downarrow}-\psi_{i \downarrow} \psi_{j \uparrow}\right\rangle\right),
\end{aligned}
$$

where not only particle-particle pairing of spinons but also their particle-hole pairing is included. In this respect the pairing symmetry of Cooper pairs has always an $s$-component although the particle-particle channel is $d$-wave. However, this quantity should not be considered to represent the true pairing symmetry of the superconducting pair. Actually, it is measured from the electron spectral function as an excitation gap, given by

$$
\begin{aligned}
& G_{i j, \uparrow \uparrow}^{e l} \equiv-\left\langle c_{i \uparrow} c_{j \uparrow}^{\dagger}\right\rangle \\
& \approx-\frac{1}{2}\left\langle e^{-i\left(\phi_{i c}-\phi_{j c}\right)}\right\rangle\left\langle\psi_{i \uparrow} \psi_{j \uparrow}^{\dagger}+\psi_{i \downarrow}^{\dagger} \psi_{j \uparrow}^{\dagger}+\psi_{i \uparrow} \psi_{j \downarrow}+\psi_{i \downarrow}^{\dagger} \psi_{j \downarrow}\right\rangle .
\end{aligned}
$$

In this respect the pairing symmetry of the superconducting order parameter will be $d-$ wave as far as the spinon pairing order parameter is $d$-wave.

\section{E. $d$-wave mean-field ansatz}

We take the $d$-wave ansatz for the pairing field $\left(Y_{i i+\hat{x}}, Y_{i i+\hat{y}}\right)=(Y,-Y)$ and uniform approximation for the hopping parameter $\left(X_{i i+\hat{x}}, X_{i i+\hat{y}}\right)=(X, X)$. The pairing potential is set $\Omega_{i}^{x, y}=0$ in the mean-field approximation because only virtual fluctuations $\left(z_{i \sigma}\right)$ are allowed due to high energy cost, while the density potential is replaced with $\Omega_{i}^{z}=-i \varphi$ for notational convenience. Introducing $b_{i c}=e^{i \phi_{i c}}$ with the rotor constraint $\left|b_{i c}\right|^{2}=1$, we write down the mean-field Lagrangian of the $\mathrm{U}(1)$ pair-rotor theory in the momentum space,

$$
\begin{aligned}
& Z=\int D\left[\psi_{k \sigma}, b_{k c}\right] e^{-\int_{0}^{\beta} d \tau L}, \\
& L=L_{\psi}+L_{\phi}+8 N t E F+N \lambda_{g}\left(X^{2}+Y^{2}-1\right), \\
& L_{\psi}=\sum_{k}\left(\begin{array}{cc}
\psi_{k \uparrow}^{\dagger} & \psi_{-k \downarrow}
\end{array}\right)\left(\begin{array}{cc}
\partial_{\tau}-\varphi & 0 \\
0 & \partial_{\tau}+\varphi
\end{array}\right)\left(\begin{array}{c}
\psi_{k \uparrow} \\
\psi_{-k \downarrow}^{\dagger}
\end{array}\right) \\
& -2 t F \sum_{k}\left(\begin{array}{ll}
\psi_{k \uparrow}^{\dagger} & \psi_{-k \downarrow}
\end{array}\right)\left(\begin{array}{cc}
X \gamma_{k} & Y \varphi_{k} \\
Y \varphi_{k} & -X \gamma_{k}
\end{array}\right)\left(\begin{array}{c}
\psi_{k \uparrow} \\
\psi_{-k \downarrow}^{\dagger}
\end{array}\right), \\
& L_{U}=\frac{1}{2 u} \sum_{k}\left[\left(i \partial_{\tau}+i \mu\right) b_{k c}\right]^{2}-4 t E Y \sum_{k} \varphi_{k} b_{k c}^{\dagger} b_{k c} \\
& +\lambda_{c} \sum_{k}\left(\left|b_{k c}\right|^{2}-1\right)+\frac{1}{2 u} \sum_{i}(i \varphi-i \mu)^{2},
\end{aligned}
$$

where $\gamma_{k}=\cos k_{x}+\cos k_{y}$ and $\varphi_{k}=\cos k_{x}-\cos k_{y} . N$ is number of lattice sites. $\lambda_{g}$ and $\lambda_{c}$ are Lagrange multiplier fields to impose the constraints for $\mathrm{SU}(2)$ gauge-matrix fields and pair-rotor fields, respectively.

Performing integration of spinon and pairon fields, we find the $\mathrm{U}(1)$ pair-rotor mean-field free energy

$$
\begin{aligned}
& F\left[b, Y, E, F, \lambda_{c}, \varphi, \mu ; \delta, T\right]=-\frac{2}{\beta} \sum_{k} \ln \left\{2 \cosh \left(\frac{\beta E_{k}^{f}}{2}\right)\right\} \\
& +\frac{1}{\beta} \sum_{q}\left[\ln \left\{2 \sinh \left(\frac{\beta}{2}\left[\mathcal{E}_{q}^{b}-\mu\right]\right)\right\}\right. \\
& \left.+\ln \left\{2 \sinh \left(\frac{\beta}{2}\left[\mathcal{E}_{q}^{b}+\mu\right]\right)\right\}\right]+N\left(8 t E F-\frac{1}{2 u}[\varphi-\mu]^{2}\right. \\
& \left.+\lambda_{c}\left[b^{2}-1\right]-\frac{\mu^{2}}{2 u} b^{2}-8 t E Y b^{2}-\mu \delta\right)
\end{aligned}
$$

where $b, \delta$, and $\beta$ are condensation amplitude, hole concentration, and inverse temperature $1 / T$, respectively. The fermion spectrum

$$
E_{k}^{f}=\sqrt{\left[2 t F \sqrt{1-Y^{2}} \gamma_{k}+\varphi\right]^{2}+\left[2 t F Y \varphi_{k}\right]^{2}}
$$

coincides with the $d$-wave BCS theory [14], and the boson spectrum is also relativistic,

$$
\mathcal{E}_{q}^{b}=\sqrt{-8 u t E Y \varphi_{q}+2 u \lambda_{c}},
$$

basically the same as the Schwinger-boson theory [8].

\section{F. Phase diagram}

It is interesting to observe that the pairon sector of the $\mathrm{U}(1)$ pair-rotor theory is almost the same as the Schwinger-boson part of the U(1) slave-fermion theory [15], where pairing correlations or antiferromagnetic fluctuations give rise to dynamics of pairons or Schwinger bosons, respectively. Actually, we find that the pairing order parameter $Y$ decreases monotonically as hole concentration increases. Since $Y$ acts as the stiffness parameter for $b$, the condensation probability $b^{2}$ is reduced (inset 
FIG. 1: As hole concentration $\delta$ increases, the superconducting transition temperature $T_{c}(\delta)$ also increases in underdoped region $(u / t=0.3)$ although the condensation amplitude $b^{2}(\delta)$ (inset) decreases.

of Fig. 1). This is basically the same as the slave-fermion theory [15] where weakening of antiferromagnetic correlations results in reduction of boson condensation. On the other hand, the superconducting transition temperature $T_{c}$, determined by vanishment of superfluid density, is shown to increase as hole concentration increases in small doping (Fig. 1).

\section{SUPERFLUID DENSITY}

\section{A. Ioffe-Larkin composition rule}

We start from the U(1) pair-rotor theory

$$
\begin{aligned}
& L_{f}=\sum_{i}\left(\begin{array}{ll}
\psi_{i \uparrow}^{\dagger} & \psi_{i \downarrow}
\end{array}\right)\left(\begin{array}{cc}
\partial_{\tau}-i a_{i \tau} & -i c_{i \tau}^{+} \\
-i c_{i \tau}^{-} & \partial_{\tau}+i a_{i \tau}
\end{array}\right)\left(\begin{array}{c}
\psi_{i \uparrow} \\
\psi_{i \downarrow}^{\dagger}
\end{array}\right) \\
& -t F \sum_{\langle i j\rangle}\left\{\left(\begin{array}{cc}
\psi_{i \uparrow}^{\dagger} & \psi_{i \downarrow}
\end{array}\right)\left(\begin{array}{cc}
X e^{i a_{i j}} & Y e^{-i c_{i j}} \\
Y e^{i c_{i j}} & -X e^{-i a_{i j}}
\end{array}\right)\left(\begin{array}{c}
\psi_{j \uparrow} \\
\psi_{j \downarrow}^{\dagger}
\end{array}\right)\right. \\
& +H . c .\}, \\
& L_{b}=\frac{1}{2 u} \sum_{i}\left(\partial_{\tau} \phi_{i c}-c_{i \tau}^{-}-i \mu\right)\left(\partial_{\tau} \phi_{i c}-c_{i \tau}^{+}-i \mu\right) \\
& -2 t E \sum_{\langle i j\rangle}\left\{2 Y \cos \left(c_{i j}\right) \cos \left(\phi_{i c}-\phi_{j c}+A_{i j}\right)\right. \\
& \left.-2 X \sin \left(a_{i j}\right) \sin \left(\phi_{i c}-\phi_{j c}+A_{i j}\right)\right\} \\
& +\frac{1}{2 u} \sum_{i}\left(a_{i \tau}+i \mu\right)^{2},
\end{aligned}
$$

where low energy fluctuations of mean-field order parameters are allowed, given by two kinds of gauge fields

$$
X_{i j}=X e^{i a_{i j}}, \quad Y_{i j}=Y e^{i c_{i j}}
$$

for their spatial components and

$$
a_{i \tau}=\Omega_{i}^{z}, \quad c_{i \tau}^{ \pm}=\Omega_{i}^{x} \mp i \Omega_{i}^{y}
$$

for their time components.

The partition function can be evaluated as a function of an electromagnetic field, expanding the effective action up to the second order for two kinds of gauge fluctuations,

$$
\begin{aligned}
& Z_{A}=\int D a_{i j} D c_{i j} D \psi_{i \sigma} D \phi_{i c} e^{-\int_{0}^{\beta} d \tau\left(L_{f}+L_{b}\right)} \\
& \approx \int D a_{i j} D c_{i j} \exp \left[-F_{M F}^{f}-F_{M F}^{b}-\frac{1}{2}\left(\frac{\partial^{2} F_{f}}{\partial a_{i j}^{2}} a_{i j}^{2}\right.\right. \\
& \left.+2 \frac{\partial^{2} F_{f}}{\partial a_{i j} c_{i j}} a_{i j} c_{i j}+\frac{\partial^{2} F_{f}}{\partial c_{i j}^{2}} c_{i j}^{2}\right)-\frac{1}{2}\left(\frac{\partial^{2} F_{b}}{\partial a_{i j}^{2}} a_{i j}^{2}+\frac{\partial^{2} F_{b}}{\partial c_{i j}^{2}} c_{i j}^{2}\right. \\
& +\frac{\partial^{2} F_{b}}{\partial A_{i j}^{2}} A_{i j}^{2}+2 \frac{\partial^{2} F_{b}}{\partial a_{i j} c_{i j}} a_{i j} c_{i j}+2 \frac{\partial^{2} F_{b}}{\partial a_{i j} A_{i j}} a_{i j} A_{i j} \\
& \left.\left.+2 \frac{\partial^{2} F_{b}}{\partial c_{i j} A_{i j}} c_{i j} A_{i j}\right)\right]
\end{aligned}
$$

where

$$
\begin{aligned}
& F_{f}\left[a_{i j}, c_{i j}\right]=-\frac{1}{\beta} \ln \int D \psi_{i \sigma} e^{-\int_{0}^{\beta} d \tau L_{f}\left[\psi_{i \sigma}, a_{i j}, c_{i j}\right]} \\
& F_{b}\left[a_{i j}, c_{i j}, A_{i j}\right]=-\frac{1}{\beta} \ln \int D \phi_{i c} e^{-\int_{0}^{\beta} d \tau L_{b}\left[\phi_{i c}, a_{i j}, c_{i j}, A_{i j}\right]}
\end{aligned}
$$

and

$$
F_{M F}^{f}=F_{f}[0,0], \quad F_{M F}^{b}=F_{b}[0,0,0]
$$

Performing the Gaussian integration for the two gauge fields, we find the partition function with an electromagnetic field

$$
\begin{aligned}
& Z_{A} \propto e^{-F_{M F}^{f}-F_{M F}^{b}} \exp \left[-\frac{1}{2}\left\{-\pi_{A A}^{b}+\frac{\pi_{a A}^{b 2}}{\pi_{a a}^{f}+\pi_{a a}^{b}}\right.\right. \\
& \left.\left.+\frac{\left(\pi_{c A}^{b}+\frac{\pi_{a c}^{f}+\pi_{a c}^{b}}{\pi_{a a}^{f}+\pi_{a a}^{b}}\right)^{2}}{\pi_{c c}^{f}+\pi_{c c}^{b}-\frac{\left(\pi_{a c}^{f}+\pi_{a c}^{b}\right)^{2}}{\pi_{a a}^{f}+\pi_{a a}^{b}}}\right\} A_{i j}^{2}\right]
\end{aligned}
$$

where $\pi_{\alpha \beta}^{f, b} \equiv-\left(\partial^{2} F_{f, b}\right) /(\partial \alpha \partial \beta)$ are current-current correlation functions with $\alpha, \beta=a, c, A$. As a result, the superfluid density is given by

$$
\rho_{s}=-\pi_{A A}^{b}+\frac{\pi_{a A}^{b 2}}{\pi_{a a}^{f}+\pi_{a a}^{b}}+\frac{\left(\pi_{c A}^{b}+\frac{\pi_{a c}^{f}+\pi_{a c}^{b}}{\pi_{a a}^{f}+\pi_{a a}^{b}}\right)^{2}}{\pi_{c c}^{f}+\pi_{c c}^{b}-\frac{\left(\pi_{a c}^{f}+\pi_{a c}^{b}\right)^{2}}{\pi_{a a}^{f}+\pi_{a a}^{b}}}
$$




\section{B. Each current-current correlation function}

The current-current correlation function can be derived as follows

$$
\begin{aligned}
& \pi_{A A}^{b} \equiv-\frac{\partial^{2} F_{b}\left[A_{i j}\right]}{\partial A_{i j}^{2}} \\
& =\frac{1}{Z_{b}} \int D \phi_{i c}\left(-\frac{\partial S_{b}}{\partial A_{i j}}\right)\left(-\frac{\partial S_{b}}{\partial A_{i j}}\right) e^{-S_{b}} \\
& -\left\{\frac{1}{Z_{b}} \int D \phi_{i c}\left(-\frac{\partial S_{b}}{\partial A_{i j}}\right) e^{-S_{b}}\right\}^{2} \\
& +\frac{1}{Z_{b}} \int D \phi_{i c}\left(-\frac{\partial^{2} S_{b}}{\partial A_{i j}^{2}}\right) e^{-S_{b}} \\
& \equiv\left\langle j_{i j}^{b A} j_{i j}^{b A}\right\rangle-\left\langle j_{i j}^{b A}\right\rangle^{2}+\left\langle K_{i j}^{b A A}\right\rangle .
\end{aligned}
$$

The first and second terms show the paramagnetic response, given by the current-current correlation function, while the last term displays the diamagnetic response, expressed by the kinetic-energy term. The "off diagonal" current-response function is given by

$$
\begin{aligned}
& \pi_{a A}^{b} \equiv-\frac{\partial^{2} F_{b}\left[a_{i j}, A_{i j}\right]}{\partial A_{i j} \partial a_{i j}} \\
& =\frac{1}{Z_{b}} \int D \phi_{i c}\left(-\frac{\partial S_{b}}{\partial A_{i j}}\right)\left(-\frac{\partial S_{b}}{\partial a_{i j}}\right) e^{-S_{b}} \\
& -\left\{\frac{1}{Z_{b}} \int D \phi_{i c}\left(-\frac{\partial S_{b}}{\partial A_{i j}}\right) e^{-S_{b}}\right\}\left\{\frac{1}{Z_{b}} \int D \phi_{i c}\left(-\frac{\partial S_{b}}{\partial a_{i j}}\right) e^{-S_{b}}\right\} \\
& +\frac{1}{Z_{b}} \int D \phi_{i c}\left(-\frac{\partial^{2} S_{b}}{\partial A_{i j} \partial a_{i j}}\right) e^{-S_{b}} \\
& \equiv\left\langle j_{i j}^{b A} j_{i j}^{b a}\right\rangle-\left\langle j_{i j}^{b A}\right\rangle\left\langle j_{i j}^{b a}\right\rangle+\left\langle K_{i j}^{b a A}\right\rangle,
\end{aligned}
$$

basically the same as the above. All other currentresponse functions are obtained in the same way as this.

Currents and kinetic terms are

$$
\begin{aligned}
j_{i j}^{b A} \equiv-\frac{\partial S_{b}}{\partial A_{i j}}=-4 t E Y \sin \left(\phi_{i c}-\phi_{j c}\right), \\
K_{i j}^{b A A} \equiv-\frac{\partial^{2} S_{b}}{\partial A_{i j}^{2}}=-4 t E Y \cos \left(\phi_{i c}-\phi_{j c}\right), \\
j_{i j}^{b a} \equiv-\frac{\partial S_{b}}{\partial a_{i j}}=-4 t E X \sin \left(\phi_{i c}-\phi_{j c}\right), \\
K_{i j}^{b a a} \equiv-\frac{\partial^{2} S_{b}}{\partial a_{i j}^{2}}=0, \quad j_{i j}^{b c} \equiv-\frac{\partial S_{b}}{\partial c_{i j}}=0, \\
K_{i j}^{b c c} \equiv-\frac{\partial^{2} S_{b}}{\partial c_{i j}^{2}}=-4 t E Y \cos \left(\phi_{i c}-\phi_{j c}\right), \\
K_{i j}^{b a A} \equiv-\frac{\partial^{2} S_{b}}{\partial A_{i j} \partial a_{i j}}=-4 t E X \cos \left(\phi_{i c}-\phi_{j c}\right), \\
K_{i j}^{b c A} \equiv-\frac{\partial^{2} S_{b}}{\partial A_{i j} \partial c_{i j}}=0, \\
K_{i j}^{b a c} \equiv-\frac{\partial^{2} S_{b}}{\partial a_{i j} \partial c_{i j}}=0
\end{aligned}
$$

for pairons and

$$
\begin{aligned}
& j_{i j}^{f a} \equiv-\frac{\partial S_{f}}{\partial a_{i j}}=-i t F X\left(\psi_{i \sigma}^{\dagger} \psi_{j \sigma}-\psi_{j \sigma}^{\dagger} \psi_{i \sigma}\right), \\
& K_{i j}^{f a a} \equiv-\frac{\partial^{2} S_{f}}{\partial a_{i j}^{2}}=-t F X\left(\psi_{i \sigma}^{\dagger} \psi_{j \sigma}+\psi_{j \sigma}^{\dagger} \psi_{i \sigma}\right), \\
& j_{i j}^{f c} \equiv-\frac{\partial S_{f}}{\partial c_{i j}}=-i t F Y\left(\psi_{i \uparrow}^{\dagger} \psi_{j \downarrow}^{\dagger}+\psi_{i \downarrow}^{\dagger} \psi_{j \uparrow}^{\dagger}\right) \\
& -i t F Y\left(\psi_{i \downarrow} \psi_{j \uparrow}+\psi_{i \uparrow} \psi_{j \downarrow}\right), \\
& K_{i j}^{f c c} \equiv-\frac{\partial^{2} S_{f}}{\partial c_{i j}^{2}}=-t F Y\left(\psi_{i \uparrow}^{\dagger} \psi_{j \downarrow}^{\dagger}-\psi_{i \downarrow}^{\dagger} \psi_{j \uparrow}^{\dagger}\right) \\
& -t F Y\left(\psi_{i \downarrow} \psi_{j \uparrow}-\psi_{i \uparrow} \psi_{j \downarrow}\right), \\
& K_{i j}^{f a c} \equiv-\frac{\partial^{2} S_{f}}{\partial a_{i j} \partial c_{i j}}=0
\end{aligned}
$$

for spinons in equilibrium of $a_{i j}, c_{i j}, A_{i j} \rightarrow 0$.

\section{Simplification in the expression of superfluid density}

Evaluating each correlation function, we find

$$
\pi_{a c}^{f}=0, \quad \pi_{a c}^{b}=0, \quad \pi_{c A}^{b}=0 .
$$

It is clear both mathematically and physically that these \}contributions should vanish. Correlations between normal and pairing currents do not exist in the spinon dynamics. Pairing-type currents do not appear in the pairon sector, causing the second and third equalities. As $(19)^{a}$ result, the expression for the superfluid density is simplified as follows

$$
\rho_{s}=-\pi_{A A}^{b}+\frac{\pi_{a A}^{b 2}}{\pi_{a a}^{f}+\pi_{a a}^{b}},
$$

similar to the conventional Ioffe-Larkin-type composition [16].

\section{Evaluation of superfluid density}

Correlation functions for superfluid density are

$$
\begin{aligned}
\pi_{A A}^{b}(q, i \Omega) & =\left\langle j_{b A}(q, i \Omega) j_{b A}(-q,-i \Omega)\right\rangle \\
& -\left\langle j_{b A}(q, i \Omega)\right\rangle\left\langle j_{b A}(-q,-i \Omega)\right\rangle+\left\langle K_{A A}^{b}(q, i \Omega)\right\rangle, \\
\pi_{a A}^{b}(q, i \Omega) & =\left\langle j_{b a}(q, i \Omega) j_{b A}(-q,-i \Omega)\right\rangle \\
& -\left\langle j_{b a}(q, i \Omega)\right\rangle\left\langle j_{b A}(-q,-i \Omega)\right\rangle+\left\langle K_{a A}^{b}(q, i \Omega)\right\rangle, \\
\pi_{a a}^{b}(q, i \Omega) & =\left\langle j_{b a}(q, i \Omega) j_{b a}(-q,-i \Omega)\right\rangle \\
& -\left\langle j_{b a}(q, i \Omega)\right\rangle\left\langle j_{b a}(-q,-i \Omega)\right\rangle, \\
\pi_{a a}^{f}(q, i \Omega) & =\left\langle j_{f a}(q, i \Omega) j_{f a}(-q,-i \Omega)\right\rangle \\
& -\left\langle j_{f a}(q, i \Omega)\right\rangle\left\langle j_{f a}(-q,-i \Omega)\right\rangle+\left\langle K_{a a}^{f}(q, i \Omega)\right\rangle
\end{aligned}
$$


in the energy-momentum space, where corresponding currents and kinetic energies are given by

$$
\begin{aligned}
& j_{b A}^{x}(q, i \Omega)=-4 t E Y \sum_{k} \sin \left(k_{x}+\frac{q_{x}}{2}\right) b_{k c}^{\dagger} b_{k+q c}, \\
& K_{A A}^{b x}(q, i \Omega)=-4 t E Y \sum_{k} \cos \left(k_{x}+\frac{q_{x}}{2}\right) b_{k c}^{\dagger} b_{k+q c} \\
& j_{b a}^{x}(q, i \Omega)=-4 t E X \sum_{k} \sin \left(k_{x}+\frac{q_{x}}{2}\right) b_{k c}^{\dagger} b_{k+q c}, \\
& K_{a A}^{b x}(q, i \Omega)=-4 t E X \sum_{k} \cos \left(k_{x}+\frac{q_{x}}{2}\right) b_{k c}^{\dagger} b_{k+q c}, \\
& j_{f a}^{x}(q, i \Omega)=2 t F X \sum_{k} \sin \left(k_{x}+\frac{q_{x}}{2}\right) \psi_{k \sigma}^{\dagger} \psi_{k+q \sigma}, \\
& K_{a a}^{f x}(q, i \Omega)=-2 t F X \sum_{k} \cos \left(k_{x}+\frac{q_{x}}{2}\right) \psi_{k \sigma}^{\dagger} \psi_{k+q \sigma} .
\end{aligned}
$$

In this expression we take the following replacement

$$
\begin{aligned}
\cos \left(\phi_{i c}-\phi_{j c}\right) & \rightarrow \frac{1}{2}\left(b_{i c}^{\dagger} b_{j c}+b_{j c}^{\dagger} b_{i c}\right) \\
\sin \left(\phi_{i c}-\phi_{j c}\right) & \rightarrow \frac{i}{2}\left(b_{i c}^{\dagger} b_{j c}-b_{j c}^{\dagger} b_{i c}\right)
\end{aligned}
$$

for evaluation of correlation functions.

Inserting Eq. (25) into Eq. (24), we find each currentcurrent correlation function in terms of each Green's function,

$$
\begin{aligned}
& \pi_{A A}^{b x x}(q, i \Omega)=16 t^{2} E^{2} Y^{2} \sum_{k} \sin \left(k_{x}+\frac{q_{x}}{2}\right) \sin \left(k_{x}-\frac{q_{x}}{2}\right) \\
& \frac{1}{\beta} \sum_{i \nu} G_{b}(k+q, i \Omega+i \nu) G_{b}(k, i \nu) \\
& +4 t E Y \frac{1}{\beta} \sum_{i \nu} \sum_{k} \cos k_{x} G_{b}(k, i \nu) \delta(q) \delta(i \Omega), \\
& \pi_{a a}^{b x x}(q, i \Omega)=16 t^{2} E^{2} X^{2} \sum_{k} \sin \left(k_{x}+\frac{q_{x}}{2}\right) \sin \left(k_{x}-\frac{q_{x}}{2}\right) \\
& \frac{1}{\beta} \sum_{i \nu} G_{b}(k+q, i \Omega+i \nu) G_{b}(k, i \nu) \\
& +4 t E X \frac{1}{\beta} \sum_{i \nu} \sum_{k} \sin k_{x} G_{b}(k, i \nu) \delta(q) \delta(i \Omega), \\
& \pi_{a A}^{b x x}(q, i \Omega)=16 t^{2} E^{2} X Y \sum_{k} \sin \left(k_{x}+\frac{q_{x}}{2}\right) \sin \left(k_{x}-\frac{q_{x}}{2}\right) \\
& \frac{1}{\beta} \sum_{i \nu} G_{b}(k+q, i \Omega+i \nu) G_{b}(k, i \nu) \\
& +4 t E X \frac{1}{\beta} \sum_{i \nu} \sum_{k} \cos k_{x} G_{b}(k, i \nu) \delta(q) \delta(i \Omega)
\end{aligned}
$$

for pairon excitations and

$$
\begin{aligned}
& \pi_{a a}^{f}(q, i \Omega)=-4 t^{2} F^{2} X^{2} \sum_{k} \sin \left(k_{x}+\frac{q_{x}}{2}\right) \sin \left(k_{x}-\frac{q_{x}}{2}\right) \\
& \frac{1}{\beta} \sum_{i \omega} \operatorname{tr}\left\{\mathbf{G}_{f}(k+q, i \Omega+i \omega) \mathbf{G}_{f}(k, i \omega)\right\} \\
& -2 t F X \frac{1}{\beta} \sum_{i \omega} \sum_{k} \cos k_{x} \operatorname{tr}\left\{\tau_{z} \mathbf{G}_{f}(k, i \omega)\right\} \delta(q) \delta(i \Omega)
\end{aligned}
$$

for spinon excitations.

The pairon propagator is

$$
\begin{aligned}
& G_{b}(q, i \Omega)=-b^{2} \delta_{q, 0} \delta_{\Omega, 0} \\
& +\frac{u}{\mathcal{E}_{q}^{b}}\left[\frac{1}{i \Omega-\mu-\mathcal{E}_{q}^{b}}-\frac{1}{i \Omega-\mu+\mathcal{E}_{q}^{b}}\right],
\end{aligned}
$$

and the spinon Nambu-propagator is

$$
\begin{aligned}
& \mathbf{G}_{f}(k, i \omega) \equiv-\left\langle\left(\begin{array}{c}
\psi_{k \uparrow} \\
\psi_{-k \downarrow}^{\dagger}
\end{array}\right)\left(\begin{array}{ll}
\psi_{k \uparrow}^{\dagger} & \psi_{-k \downarrow}
\end{array}\right)\right\rangle \\
& \equiv\left(\begin{array}{cc}
G_{f}(k, i \omega) & F(k, i \omega) \\
F^{*}(k, i \omega) & -G_{f}^{*}(k, i \omega)
\end{array}\right),
\end{aligned}
$$

where the normal Green's function is

$$
G_{f}(k, i \omega)=\frac{1}{2}\left[\frac{1-\frac{\varphi+2 t F X \gamma_{k}}{E_{k}^{f}}}{i \omega-E_{k}^{f}}+\frac{1+\frac{\varphi+2 t F X \gamma_{k}}{E_{k}^{f}}}{i \omega+E_{k}^{f}}\right]
$$

and the anomalous propagator

$$
F(k, i \omega)=-\frac{t F Y \varphi_{k}}{E_{k}^{f}}\left[\frac{1}{i \omega-E_{k}^{f}}-\frac{1}{i \omega+E_{k}^{f}}\right]
$$

Inserting these Green's functions into Eqs. (26) and (27), and performing the Matsubara frequency summation, we obtain final expressions for all current-current correlation 
functions in the static limit,

$$
\begin{aligned}
& \pi_{A A}^{b x x}(q \rightarrow 0, i \Omega=0) \\
& \approx 16 t^{2} E^{2} Y^{2} u^{2} \sum_{k} \frac{\sin ^{2} k_{x}}{\mathcal{E}_{k}^{b 2}}\left\{\left(-\frac{\partial n\left(\mathcal{E}_{k}^{b}+\mu\right)}{\partial\left[\mathcal{E}_{k}^{b}+\mu\right]}\right)\right. \\
& \left.+\frac{n\left(\mathcal{E}_{k}^{b}+\mu\right)-n\left(-\mathcal{E}_{k}^{b}+\mu\right)}{\mathcal{E}_{k}^{b}}\right\}-4 t E Y b^{2} \\
& -4 t E Y u \sum_{k} \frac{\cos k_{x}}{\mathcal{E}_{k}^{b}}\left[n\left(\mathcal{E}_{k}^{b}+\mu\right)-n\left(-\mathcal{E}_{k}^{b}+\mu\right)\right], \\
& \pi_{a a}^{b x x}(q \rightarrow 0, i \Omega=0) \\
& \approx 16 t^{2} E^{2} X^{2} u^{2} \sum_{k} \frac{\sin ^{2} k_{x}}{\mathcal{E}_{k}^{b 2}}\left\{\left(-\frac{\partial n\left(\mathcal{E}_{k}^{b}+\mu\right)}{\partial\left[\mathcal{E}_{k}^{b}+\mu\right]}\right)\right. \\
& \left.+\frac{n\left(\mathcal{E}_{k}^{b}+\mu\right)-n\left(-\mathcal{E}_{k}^{b}+\mu\right)}{\mathcal{E}_{k}^{b}}\right\} \\
& -4 t E X u \sum_{k} \frac{\sin _{k x}}{\mathcal{E}_{k}^{b}}\left[n\left(\mathcal{E}_{k}^{b}+\mu\right)-n\left(-\mathcal{E}_{k}^{b}+\mu\right)\right], \\
& \pi_{a A}^{b x x}(q \rightarrow 0, i \Omega=0) \\
& \approx 16 t^{2} E^{2} X Y u^{2} \sum_{k} \frac{\sin ^{2} k_{x}}{\mathcal{E}_{k}^{b 2}}\left\{\left(-\frac{\partial n\left(\mathcal{E}_{k}^{b}+\mu\right)}{\partial\left[\mathcal{E}_{k}^{b}+\mu\right]}\right)\right. \\
& \left.+\frac{n\left(\mathcal{E}_{k}^{b}+\mu\right)-n\left(-\mathcal{E}_{k}^{b}+\mu\right)}{\mathcal{E}_{k}^{b}}\right\}-4 t E X b^{2} \\
& -4 t E X u \sum_{k} \frac{\cos k_{x}}{\mathcal{E}_{k}^{b}}\left[n\left(\mathcal{E}_{k}^{b}+\mu\right)-n\left(-\mathcal{E}_{k}^{b}+\mu\right)\right]
\end{aligned}
$$

for pairons and

$$
\begin{aligned}
& \pi_{a a}^{f}(q \rightarrow 0, i \Omega=0) \\
& \approx-4 t^{2} F^{2} X^{2} \sum_{k} \sin ^{2} k_{x}\left(-\frac{\partial f\left(E_{k}^{f}\right)}{\partial E_{k}^{f}}\right) \\
& -2 t F X \sum_{k} \cos k_{x}\left(\frac{\varphi+2 t F X \gamma_{k}}{E_{k}^{f}}\right) \tanh \left(\frac{\beta E_{k}^{f}}{2}\right)
\end{aligned}
$$

for spinons, where the spinon contribution is basically the same as that of the BCS theory [14]. Inserting Eqs. (31) and (32) into Eq. (23), we find the superfluid density as a function of hole concentration and temperature in the $\mathrm{U}(1)$ pair-rotor mean-field theory.
FIG. 2: (Color online) The decreasing ratio of superfluid density $\rho_{s}(T)$ is enhanced as hole concentration is reduced, where $\delta=0.01$ (solid), $\delta=0.03$ (dashed), $\delta=0.05$ (dash$\operatorname{dot}$ ), $\delta=0.07$ (dash-dot-dot), and $\delta=0.09$ (dotted).

\section{E. Superfluid density as a function of hole concentration and temperature}

We find that the dominant contribution is given by

$$
\begin{aligned}
& \rho_{s}(T) \approx-\pi_{A A}^{b x x}(q \rightarrow 0, i \Omega=0 ; T) \\
& =4 t E Y b^{2}+4 t E Y u \sum_{k} \frac{\cos k_{x}}{\mathcal{E}_{k}^{b}}\left[n\left(\mathcal{E}_{k}^{b}+\mu\right)-n\left(-\mathcal{E}_{k}^{b}+\mu\right)\right] \\
& -16 t^{2} E^{2} Y^{2} u^{2} \sum_{k} \frac{\sin ^{2} k_{x}}{\mathcal{E}_{k}^{b 2}}\left\{\left(-\frac{\partial n\left(\mathcal{E}_{k}^{b}+\mu\right)}{\partial\left[\mathcal{E}_{k}^{b}+\mu\right]}\right)\right. \\
& \left.+\frac{n\left(\mathcal{E}_{k}^{b}+\mu\right)-n\left(-\mathcal{E}_{k}^{b}+\mu\right)}{\mathcal{E}_{k}^{b}}\right\},
\end{aligned}
$$

where the first two terms are diamagnetic contributions and the last term is paramagnetic. This expression is simplified as

$$
\rho_{s}(T) \equiv \rho_{s}+\left(\frac{d \rho_{s}(T)}{d T}\right)_{T \rightarrow 0} T,
$$

where the zero temperature superfluid density is $\rho_{s} \approx$ $4 t E Y b^{2}$ and the decreasing ratio is $\left(\frac{d \rho_{s}(T)}{d T}\right)_{T \rightarrow 0} \approx$ $\frac{1}{\Lambda^{2}} \ln \left(-\frac{2 \Lambda \sqrt{u t E Y}}{\mu}\right)$ with momentum cutoff $\Lambda$.

Fig. 2 shows the superfluid density with various hole doping. Interestingly, the decreasing ratio of the superfluid density is enhanced as hole concentration is reduced, giving rise to the monotonically increasing $T_{c}(\delta)$ in Fig. 1. We interpret this tendency as the realization of the density-phase uncertainty principle because phase fluctuations of Cooper pairs are stronger in small doping.

We would like to point out that reduction of the superfluid density originates from phase fluctuations in the $\mathrm{U}(1)$ pair-rotor theory instead of scattering with Dirac fermions [17]. The contributions of Dirac fermions also result in the temperature-linear decreasing ratio. However, such contributions become irrelevant in the IoffeLarkin expression, resulting from non-minimal coupling to gauge fields in the pairon sector. 


\section{DISCUSSION}

\section{A. Comparison with the BCS theory}

It is interesting to observe that the $\mathrm{U}(1)$ pair-rotor theory [Eq. (14)] is almost "dual" to the slave-fermion theory [15], where the charge $\mathrm{SU}(2)$ symmetry is replaced with the spin $\mathrm{SU}(2)$ symmetry. In this respect the pseudogap state, where Cooper pairs are not coherent globally, is a mirror image of the so called anomalous metal phase, sometimes referred as the algebraic charge liquid in the slave-fermion description [15, 18, 19], where antiferromagnetic correlations exist only locally. Emergence of such an anomalous state reflects strong quantum fluctuations, based on the uncertainty principle.

It is important to notice that the $\mathrm{SU}(2)$ slave-rotor representation is difficult to be applied to the negativeinteraction model because the time-fluctuation term in the rotor Lagrangian of Eq. (2) becomes negative, thus its path-integral representation is not defined consistently. This implies that the $\mathrm{U}(1)$ pair-rotor theory differs from the BCS theory in itself.

\section{B. Origin of spectral asymmetry}

We show that the spectral asymmetry [20] appears naturally in the $\mathrm{U}(1)$ pair-rotor theory. The electron Green's function is given by multiplication of the boson [Eq. (28)] and fermion [Eqs. (29) and (30)] Green's functions, $G_{x x^{\prime}}^{e l} \approx-G_{x x^{\prime}}^{b}\left(G_{x x^{\prime}}^{f}+F_{x x^{\prime}}^{*}+F_{x x^{\prime}}-G_{x x^{\prime}}^{f *}\right)$, where $F_{x x^{\prime}}$ is an anomalous Green's function due to pairing. Then, we obtain the spectral intensity $A_{e l}(k, \omega)=$ $A_{\text {coh }}(k, \omega)+A_{\text {in }}(k, \omega)$, where $A_{i n}(k, \omega)$ is an incoherent background and the coherent part is

$$
\begin{aligned}
& A_{c o h}(k, \omega)=b^{2}\left\{\left(1-\frac{2 t F Y \varphi_{k}}{E_{k}^{f}}\right) \delta\left(\omega-E_{k}^{f}\right)\right. \\
& \left.+\left(1+\frac{2 t F Y \varphi_{k}}{E_{k}^{f}}\right) \delta\left(\omega+E_{k}^{f}\right)\right\},
\end{aligned}
$$

showing the spectral asymmetry which originates from pairing correlations. This predicts that the spectral asymmetry will disappear when pairing correlations vanish at a temperature, usually identified with the pseudogap temperature $T^{*}$. More quantitative analysis is necessary.

\section{Application of the SU(2) slave-rotor theory to one dimension}

It is valuable to apply the $\mathrm{SU}(2)$ slave-rotor representation to the one-dimensional Hubbard model. Actu- ally, one has the same $\mathrm{SU}(2)$ slave-rotor Lagrangian as Eq. (2) in one dimension. Considering that the fermion part is an $\mathrm{SU}(2)$ gauge theory in $(1+1) D$, non-Abelian bosonization of $\mathrm{QCD}_{2}$ results in the $\mathrm{SU}_{k=1}(2)$ WZNW (Wess-Zumino-Novikov-Witten) theory [21] with level $k$. Combining this fermion sector with the pairon part, we obtain the $\mathrm{SO}(4)$ WZNW theory for spin dynamics and $\mathrm{SO}(4)$ nonlinear $\sigma$ model without the topological term for charge dynamics at half filling $(\mu=0)$, where spin dynamics is decoupled from charge dynamics [21]. As a result, charge fluctuations are gapped, corresponding to a Mott insulator, while spin excitations are critical due to the presence of the topological term. Hence charge fluctuations are described by the pair-rotor Lagrangian even in one dimension, implying that our formulation generalizes the bosonization scheme of one dimensional charge dynamics.

\section{CONCLUSION}

In this paper we proposed a mechanism of superconductivity based on the $\mathrm{U}(1)$ pair-rotor theory, where quantum fluctuations for phase dynamics of Cooper pairs are taken into account. An important feature is that the single energy scale for the Cooper pair formation and phase coherence is separated into two energy scales, allowing the pseudogap phase, where quantum phase fluctuations are so strong as to destroy the superconductivity, but superconducting correlations still exist at least locally. We argued that emergence of such two energy scales is the hallmark of the strong coupling approach as the Schwinger-boson theory for antiferromagnetism of localized spins where the spin-gap phase corresponds to the pseudogap state, differentiated from the weak coupling approach such as the BCS theory [5] or HMM framework 7].

\section{Acknowledgments}

K.-S. Kim thanks J.-H. Han for helpful discussions. K.S. Kim was supported by the National Research Foundation of Korea (NRF) grant funded by the Korea government (MEST) (No. 2009-0074542). 
[1] P. Monthoux, D. Pines, and G. G. Lonzarich, Nature 450, 1177 (2007).

[2] A. T. Zheleznyak, V. M. Yakovenko, and I. E. Dzyaloshinskii, Phys. Rev. B 55, 3200 (1997).

[3] A. V. Chubukov, D. V. Efremov, and I. Eremin, Phys. Rev. B 78, 134512 (2008).

[4] Ar. Abanov, A. V. Chubukov, and J. Schmalian, Adv. Phys. 52, 119 (2003); A. V. Chubukov, D. Pines, and J. Schmalian, in The Physics of Superconductors, edited by K. H. Bennemann and J. B. Ketterson (Springer, New York 2003), Vol. 1, p. 495.

[5] J. R. Schrieffer, Theory of Superconductivity (Westview Press, 1999).

[6] J. Rech, C. Pépin, and A. V. Chubukov, Phys. Rev. B 74, 195126 (2006).

[7] T. Moriya and J. Kawabata, J. Phys. Soc. Jpn. 34, 639 (1973); T. Moriya and J. Kawabata, J. Phys. Soc. Jpn. 35, 669 (1973); J. A. Hertz, Phys. Rev. B 14, 1165 (1976); A. J. Millis, Phys. Rev. B 48, 7183 (1993).

[8] A. Auerbach, Interacting Electrons and Quantum magnetism (Springer-Verlag, 1994).

[9] K.-S. Kim, Phys. Rev. Lett. 97, 136402 (2006); Phys. Rev. B 75, 245105 (2007).

[10] P. W. Anderson, The Theory of Superconductivity in the High Tc Cuprates (Princeton University Press, Princeton 1997).

[11] P. A. Lee, N. Nagaosa, and X.-G. Wen, Rev. Mod. Phys.
78, 17 (2006).

[12] R. G. Leigh, P. Phillips, and T.-P. Choy, Phys. Rev. Lett. 99, 046404 (2007); T.-P. Choy, R. G. Leigh, P. Phillips, and P. D. Powell, Phys. Rev. B 77, 014512 (2008).

[13] S. Florens and A. Georges, Phys. Rev. B 70, 035114 (2004); E. Zhao and A. Paramekanti, Phys. Rev. B 76, 195101 (2007).

[14] A. C. Durst and P. A. Lee, Phys. Rev. B 62, 1270 (2000).

[15] Chenglong Jia and K.-S. Kim, arXiv:0811.2626 (unpublished).

[16] L. B. Ioffe and A. I. Larkin, Phys. Rev. B 39, 8988 (1989); L. B. Ioffe and G. Kotliar, Phys. Rev. B 42, 10348 (1990); P. A. Lee and N. Nagaosa, Phys. Rev. B 46, 5621 (1992).

[17] D.-H. Lee, Phys. Rev. Lett. 84, 2694 (2000); P. A. Lee, Physica C 317-318, 194 (1999).

[18] R. K. Kaul, Y. B. Kim, S. Sachdev, and T. Senthil, Nature Physics 4, 28 (2008).

[19] K.-S. Kim and M. D. Kim, Phys. Rev. B 77, 125103 (2008); Phys. Rev. B 75, 035117 (2007).

[20] M. Randeria, R. Sensarma, N. Trivedi, and F.-C. Zhang, Phys. Rev. Lett. 95, 137001 (2005), and references therein.

[21] A. O. Gogolin, A. A. Nersesyan, and A. M. Tsvelik, Bosonization and Strongly Correlated Systems (Cambridge University Press, Cambridge, 2004). 